\title{
Ten-year advances in immunopathology of glomerulonephritis: translated into patients' care or lost in translation?
}

\author{
Pierre Ronco • Jürgen Floege
}

Received: 6 June 2014 / Accepted: 6 June 2014 / Published online: 24 July 2014

(C) Springer-Verlag Berlin Heidelberg 2014

Because substantial advances have occurred in the understanding of the pathomechanisms of immune-mediated glomerulonephritis in the last 10 years, we thought that the time had come for an update. The seven articles gathered in this special issue of the Seminars in Immunology and Immunopathology do not pretend to be exhaustive but are aimed at shedding light on the most fascinating breakthroughs that occurred in the field. But what has really been achieved that can be translated in practical terms for the pathologists, the clinicians, and more importantly for the patients? Where do the advances modify diagnostic tests and classification of diseases leading to a new ontology? What is the impact on therapeutic strategies? What are the hopes and what are the threats?

There are two areas where the bidirectional translation from the bench to the bedside has been incredibly fast, that is membranous nephropathy (MN) and atypical hemolytic uremic syndrome (aHUS). MN actually is a success story of translational research where a highly reproducible experimental model described by a pediatrician - Walter Heymann - in the late 1950s finally led to understanding the human disease with the discovery of anti-PLA2R antibodies by Beck et al. $[1,2]$. Major steps in between were the demonstration that immune complexes were formed in situ in the glomerular capillary wall [3, 4]; the identification of megalin, the podocyte target antigen of circulating antibodies by

This article is a contribution to the special issue on Immunopathology of Glomerular Diseases - Guest Editors: P. Ronco and J. Floege

\section{P. Ronco $(\bowtie)$}

Hopital Tenon, Paris, France

e-mail: pierreronco@yahoo.fr

\section{J. Floege $(\bowtie)$}

Division of Nephrology, RWTH Aachen University Hospital,

Pauwelsstr. 30, 52057 Aachen, Germany

e-mail: juergen.floege@rwth-aachen.de
Kerjaschki and Farquhar [5]; and the characterization of the first human podocyte antigen in a neonate born with alloimmune MN by Debiec et al. [6] who provided the proof of concept that also in humans, a podocyte antigen could serve as a target for pathogenic antibodies and who laid the ground for the identification of PLA2R in 2009. Less than 4 years later, both an immunofluorescence test and an ELISA are commercially available. The specificity of anti-PLA2R for MN, close to $100 \%$, is such that indications of kidney biopsies in antiPLA2R-positive patients with a nephrotic syndrome may have to be revisited. Next to their diagnostic value, anti-PLA2R antibodies seem to be valuable tools for predicting outcome and monitoring activity of $\mathrm{MN}$ and consequently immunosuppressive therapy although further studies in extensively phenotyped prospective cohorts are needed [2].

Another area where research advances have had a major impact on therapy is aHUS [7]. Although aHUS is a very rare disease, genetic advances fueled by careful clinical observations have enlightened our understanding not only of the pathogenesis of the disease but also of other clinical conditions such as HUS occurring during pregnancy or postpartum and glomerulonephritis with prevailing deposits of C3. Even more importantly, the identification of inherited or acquired alterations of the alternative pathway of complement activation has resulted in new therapies based on the use of anti-C5 antibody. The association between aHUS and low complement factor $\mathrm{H}$ levels (CFH) had been described since the 1980s, but it was only in 1998 that advances in the genetic tools enabled the identification of the first mutation in the $\mathrm{CFH}$ gene in aHUS by Warwicker et al. [8]. Since then, mutations have been identified in five other genes of the alternative complement pathway, in the thrombomodulin and diacylglycerol kinase epsilon genes, while antibodies to factor $\mathrm{H}$ were first identified in 2005 by DragonDurey et al. [9]. To date, one or more genetic or acquired abnormalities in the complement system have been documented 
in nearly $60 \%$ of patients with aHUS [7]. Complement abnormalities may also contribute to HUS induced by Shiga-like toxin-producing bacteria. Therapeutic translation of these ground-breaking findings was spectacular in aHUS even if a number of questions remain. Because the activation of the terminal complement pathway is essential for the development of the endothelial lesion that characterizes aHUS, eculizumab, previously used for paroxysmal nocturnal hemoglobinuria, was approved by the European Medicine Agency (EMA) and by the US Food and Drug Administration (FDA) in 2011 for the treatment of aHUS after successful trials in adults and adolescents [10]. Although the duration of administration and ideal protocols taking in account the unaffordably high cost of the drug, eculizumab represents a paradigm change in the treatment of aHUS patients. This therapeutic revolution also applies to aHUS patients receiving a kidney graft as recurrence occurs in 64 to $78 \%$ of transplanted patients with $\mathrm{CFH}, \mathrm{CFI}$, and $\mathrm{C} 3$ mutations, and graft failure occurred in 75 to $95 \%$ of them [7]. Preliminary studies indicate that eculizumab can prevent and treat post-transplant recurrence although further trials are required.

It is clear that the immunopathologic advances described above have led to break down MN and aHUS into different immunologically or genetically defined entities. It does not suffice any longer to describe a disease only clinically and pathologically, but immunologic (anti-PLA2R antibodies, anti$\mathrm{CFH}$ antibodies) and genetic (mutations) findings are essential to the definition, the classification (ontology), and the treatment. This is well illustrated by the other glomerular diseases dealt with in this issue of Seminars in Immunology and Immunopathology.

Although substantial progresses have been made in understanding the immunopathology of minimal change disease (MCD) [11], IgA nephropathy [12], ANCA-related vasculitis [13], and lupus nephritis [14], they have not yet translated into major diagnostic and treatment improvements. The nature of the putative circulating permeability factor(s) in MCD and FSGS is still elusive despite some clues provided by works on podocyte-secreted angiopoietin-like 4 [15] and, more controversial, suPAR, the soluble urokinase-type plasminogen activator receptor [16]. Recent data indicate that abatacept, a costimulatory inhibitor that targets CD80/B7-1, might be efficient in some B7-1-positive proteinuric diseases particularly FSGS [17] although these data led to controversial comments and will need independent confirmation. In IgA nephropathy $(\operatorname{IgAN})$, a key pathogenic role appears to be played by underglycated $\operatorname{IgA} 1$ as well as autoantibodies to these $\operatorname{IgA}$ glycoforms and $\operatorname{IgA}$ receptors such as CD89 and transferrin receptor 1 . However, studies are made difficult by the lack of good animal models of IgAN, the heterogeneity of the disease, and it has now become clear that the IgAN cases coming to clinical attention are only the tip of the iceberg. Particular attention is therefore being paid to secondary effector mechanisms occurring after the deposition of IgA containing immune complexes including complement activation, release of mesangial growth factors (in particular platelet-derived growth factor), and non-IgAN-specific events that culminate in glomerular and subsequently renal tubulointerstitial scarring. IgAN heterogeneity is well reflected by the many loci that were identified in pangenomic studies [12] while only 2 loci, HLADQA1 and PLA2R1, showed up in similar studies of Caucasian patients with idiopathic MN [18] which is a much more homogeneous disease. In lupus nephritis, there is a double level of complexity as both extrarenal (the complex combination of genetic variants and environmental factors that lead to SLE) and intrarenal pathomechanisms contribute to the immunopathology. Antibodies bind to intrarenal nuclear autoantigens or annexins, which activates complement and $\mathrm{Fc}$ receptors. This process drives the various types of immune complex glomerulonephritis, which depends on the predominant intraglomerular compartment where the immune complexes form. Overall, these researches have not translated into appreciable therapeutic improvement mostly because one faces several diseases hiding under the same denomination (IgAN) or at least several different mechanisms (lupus nephritis). As it has been alluded before, these observations call for a new classification of diseases based on pathophysiology. Results of the pangenomic studies of patients with ANCArelated vasculitis strongly support this statement since these studies clearly showed an association of PR3-associated vasculitis with HLA class II, proteinase 3, and alpha-1 antitrypsin while no predisposing variant was identified in the case of MPO-associated vasculitis [13].

In cancer therapy, personalized treatment based on an extensive characterization of the tumor cells both in the primary tumor and the metastases has become the rule [19], and several lines of treatment according to the evolution of markers are proposed. In contrast, treatment of immune-mediated GN has remained very empirical and still relies to a large extent on a single line of nonspecific therapies such as corticosteroids and immunosuppressive drugs which are more or less the same since more than 30 years. The only two notable exceptions are eculizumab and rituximab. It is interesting to note that the latter anti-B cell therapy was initially used in B cell lymphomas and leukemias. We are still far from therapies targeting specific mediators at different stages of the nephropathy for several reasons. First, immune-mediated glomerular diseases are rare, and clinical trials are all undersized. Second, we still lack good biomarkers such as hormonal receptors, growth factor receptors, or oncogene rearrangements or mutations. This is well illustrated by research on the mechanisms of crescent formation [20]. There are still uncertainties regarding the nature of proliferating cells, the interplay of mediators, and the relevance of experimental models. Furthermore, experiments in crescentic GN are often targeted at a single mediator or pathway with often spectacular results of pharmacological orgeneticinhibition. However, who 
can believe that such complex diseases involve a single actor? The recent approach of integrative biology applied to renal diseases does suggest the opposite while revealing unexpected commonalities between non-immune diabetic nephropathy and immune-mediated GN $[21,22]$. Would we treat a cancer with a single drug? Considerable efforts should be devoted to a better characterization of immune-mediated GN, to the use of combined therapy in well-defined experimental models, and to the screening of compound libraries for the repurposing of already approved drugs in other non-renal diseases.

As very appropriately pointed out by William G. Couser in a recent review of basic and translational concepts of immunemediated glomerular diseases [23], three ingredients have remained constant over the years: the patients, the need for well-characterized relevant models of their diseases, and the contributions of physician-scientists who have accounted for most of the advances described in this issue. The patients and the need for animal models will remain, but the technology to study them will exponentially advance in the next decade. However, clinical investigators are threatened by overload of clinical work due to heavy financial constrains affecting hospital staffs in the Western world and by shortage of funding although both are not restricted to nephrology. Continued progress in the area of renal immunopathology will require the availability and dedication of investigators who fully understand both the tools of basic science and the clinical and pathologic manifestations of human renal diseases as well as their opening to other specialties such as oncology and hematology where progress has undoubtedly been faster.

Have the exciting data presented in this issue of the Seminars in Immunology and Immunopathology translated into appreciable advances for the clinicians and the patients or have they been lost in translation? Response to this important question varies mostly depending on the glomerular disease, its heterogeneity, and its complexity. We believe that all the ingredients of success are available, but perhaps a change in the way we approach complexity and a wider opening toward more advanced disciplines would make our projects more successful in terms of translation for our patients.

Acknowledgments We thank all our well-recognized experts for their scholarly contributions and hope their reviews will be highly stimulating, particularly in the ways discussed above.

\section{References}

1. Beck LH Jr, Bonegio RG, Lambeau G et al (2009) M-type phospholipase A2 receptor as target antigen in idiopathic MN. N Engl J Med 361:11-21

2. Debiec H, Ronco P (2014) Immunopathogenesis of membranous nephropathy: an update. Semin Immunopathol. doi:10.1007/ s00281-014-0423-y (this issue)
3. Van Damme BJ, Fleuren GJ, Bakker WW et al (1978) Experimental glomerulonephritis in the rat induced by antibodies directed against tubular antigens. V. Fixed glomerular antigens in the pathogenesis of heterologous immune complex glomerulonephritis. Lab Invest 38: $502-510$

4. Couser WG, Steinmuller DR, Stilmant MM et al (1978) Experimental glomerulonephritis in the isolated perfused rat kidney. $\mathrm{J}$ Clin Invest 62:1275-1287

5. Kerjaschki D, Farquhar M (1982) The pathogenic antigen of Heymann nephritis is a membrane glycoprotein of the renal proximal tubule brush border. Proc Natl Acad Sci 79:55575561

6. Debiec H, Guigonis V, Mougenot B et al (2002) Antenatal membranous glomerulonephritis due to anti-neutral endopeptidase antibodies. N Engl J Med 346:2053-2060

7. Mele C, Remuzzi G, Noris M (2014) Hemolytic uremic syndrome. Semin Immunopathol. doi:10.1007/s00281-014-0416-x (this issue)

8. Warwicker P, Goodship TH, Donne RL et al (1998) Genetic studies into inherited and sporadic hemolytic uremic syndrome. Kidney Int 53:836-844

9. Dragon-Durey MA, Loirat C, Cloarec S et al (2005) Anti-factor H autoantibodies associated with atypical hemolytic uremic syndrome. J Am Soc Nephrol 16:555-563

10. Legendre CM, Licht C, Muus P et al (2013) Terminal complement inhibitor eculizumab in atypical hemolytic-uremic syndrome. N Engl J Med 368:2169-2181

11. Sahali D, Sendeyo K, Mangier M et al (2013) Immunopathogenesis of idiopathic nephrotic syndrome with relapse. Semin Immunopathol. doi:10.1007/s00281-013-0415-3 (this issue)

12. Floege J, Moura IC, Daha MR (2014) New insights into the pathogenesis of IgA nephropathy. Semin Immunopathol. doi:10.1007/ s00281-013-0411-7 (this issue)

13. McKinney EF, Willcocks LC, Broecker V, Smith KGC (2014) The immunopathology of ANCA-associated vasculitis. Semin Immunopathol. doi:10.1007/s00281-014-0436-6 (this issue)

14. Anders H-J, Fogo AB (2013) Immunopathology of lupus nephritis. Semin Immunopathol. doi:10.1007/s00281-013-0413-5 (this issue)

15. Clement LC, Avila-Casado C, Macé C et al (2011) Podocyte-secreted angiopoietin-like-4 mediates proteinuria in glucocorticoid-sensitive nephrotic syndrome. Nat Med 17:117-122

16. Reiser J, Chapman H (2014) Soluble urokinase-type plasminogen activator receptor in FSGS: stirred but not shaken. J Am Soc Nephrol(May)

17. Yu CC, Fornoni A, Weins A et al (2013) Abatacept in B7-1positive proteinuric kidney disease. N Engl J Med 369:24162423

18. Stanescu HC, Arcos-Burgos M, Medlar A et al (2011) Risk HLADQA1 and PLA(2)R1 alleles in idiopathic membranous nephropathy. N Engl J Med 364:616-626

19. De Abreu FB, Wells WA, Tsongalis GJ (2013) The emerging role of the molecular diagnostics laboratory in breast cancer personalized medicine. Am J Pathol 183:1075-1083

20. Hénique C, Papista C, Guyonnet L et al (2014) Update on crescentic glomerulonephritis. Semin Immunopathol. doi:10.1007/s00281-0140435-7 (this issue)

21. Schmid H, Boucherot A, Yasuda Y et al (2006) Modular activation of nuclear factor-kappaB transcriptional programs in human diabetic nephropathy. Diabetes 55:2993-300

22. Kretzler M, Cohen CD (2010) Integrative biology of renal disease: toward a holistic understanding of the kidney's function and failure. Semin Nephrol 3:439-442

23. Couser WG (2012) Basic and translational concepts of immune-mediated glomerular diseases. J Am Soc Nephrol 23:381-399 